\title{
A pilot study of Korean Medical Examination
}

\author{
Junghun Yoo ${ }^{1}$, Sanghun Lee ${ }^{1,2}$, Soyoung Kim ${ }^{1,2}$, Daehyeok Kim ${ }^{1}$, Jeong Hwan Park, \\ ${ }^{1} \mathrm{KM}$ Data Division, Korea Institute of Oriental Medicine \\ ${ }^{2}$ University of Science \& Technology (UST), Korean Convergence Medicine
}

\begin{abstract}
Objectives: This study aimed to evaluate the feasibility of the Korean medical examination protocol, which included 14 questionnaires and 20 medical examination devices.

Methods: We conducted a pilot observational study of 90 subjects to measure the time required to fulfill each item of the Korean medical examination, to evaluate patient satisfaction, and to report improvements that can be made to the Korean medical examination protocol based on clinical coordinator and subject feedback.

Results: Among the 90 subjects included in the study (59 women and 31 men; mean [standard deviation] age, 37.2 [12.3] years), over $80 \%$ intended to receive a Korean medical examination if hospitals provided it and would recommend a Korean medical examination to others. The average time spent on the overall Korean medical examination was approximately 88.0 (21.4) minutes. Three areas for improvements were reported: survey issues, including the number of items, understanding, and readability; error issues in device measurements; and environmental issues affecting the sequence of medical examinations and temperature.

Conclusions: Most subjects were satisfied with the Korean medical examination. Future studies should be conducted with larger samples to collect data continuously.
\end{abstract}

\section{$\overline{\text { Key Words }}$ : Korean medical examination, Feasibility, Observation study}

\section{Introduction}

A medical examination is the process of screening people who are likely to have diseases that have not yet significantly progressed, by conducting specific medical tests on patients who have seemingly no symptoms ${ }^{1}$. Korea has been conducting national medical examinations since 1995 , with a screening rate of over $70 \%$ over the past five years, accumulating medical examination data from over 10 million individuals every year ${ }^{2}$. The National Health Insurance Service established a medical examination cohort with over 500,000 cases and shared this data with other researchers to actively conduct further studies ${ }^{3)}$.

Prior studies of the Korean Medical Examination (KME) included the development of questionnaires and $\operatorname{program}^{4,5)}$, satisfaction survey ${ }^{9,10)}$, and association analysis ${ }^{6-8)}$. For example, Moon et al. developed medical examination questionnaires and a software based on diagnosis as a tool for evaluating medical health in the Korean population $^{4)}$. In addition, Lee et al. described the contents and patient satisfaction of the KME

\footnotetext{
- Received : 15 January 2021

- Revised : 21 June 2021

- Accepted : 5 November 2021

- Correspondence to : Jeong Hwan Park

KM Data Division, Korea Institute of Oriental Medicine

1672 Yuseong-daero, Yuseong-gu, Daejeon, 34054, Republic of Korea

Tel : +82-42-868-9358, E-mail : siegfriegd@kiom.re.kr
} 
program in 1,567 subjects and compared the characteristics of the Korean health examination with that of the Western health examination". The association between physical activity and health factors such as stress and fatigue was analyzed in the Korean health examination for 20,509 workers ${ }^{8)}$.

A growing body of research has collected objective data on Korean medicine. Diagnosis in Korean medicine, which used to be measured based on the doctor's subjective opinion, was quantified and objectively assessed by developing devices and questionnaires. For example, a study reported that pulse diagnosis was developed from devices using one piezoelectric sensor to measure derivative pressure pulse waveforms in 1968 to devices detecting three-dimensional waveforms to quantify the expended pressure in $2003^{11)}$. In addition, various models of algometers were used to quantitatively measure the pressure pain threshold during an abdominal examination, and the measurement protocol was standardized to ensure reproducibility ${ }^{12}$. Further, a standardized questionnaire to measure seven emotions in Korean medicine was developed by expert recommendations, internal consistency and reliability analysis, test-retest reliability analysis, factor analysis, and correlation analysis $^{13)}$.

We attempted to build a large data infrastructure through the KME protocol, which uses standardized and quantified devices and questionnaires. We conducted this pilot observational study to evaluate the feasibility of the KME protocol as the first step for a large-scale study.

\section{Methods}

\section{Study design}

This was a pilot observational study that evaluated the feasibility of the KME protocol. The KME protocol was developed by standardizing and quantifying the measurement protocol through the following steps. First, candidates for clinical parameters of Korean medicine were extracted from the literature review. Second, a committee of 15 experts recommended by the Association of Korean Medicine and each branch institute selected key parameters by scoring the suitability of the KME items and its clinical importance. Third, to measure parameters objectively, questionnaires were structured, and devices were chosen. Lastly, the protocol and SOPs of the final 14 questionnaires and 20 medical examinations were developed.

Questionnaires consisted of 10 clinical questionnaires and 4 previously developed questionnaires. Participants were asked to complete 10 clinical questionnaires on the following items: thirst/ xerostomia/amount of water consumed, stool, urine, waking up, heat and cold patterns, eating habits, alcohol habits, smoking habits, exercise habits, and menstrual health. The Pittsburgh Sleep Quality Index (PSQI) was used to measure the quality of sleep ${ }^{14,15)}$. Perceived stress was evaluated using the 10-item Perceived Stress Scale (PSS) 16,17). The Core Seven Emotions Inventory (CSEI), consisting of 100 items, was used to assess the key emotions considered as a cause of disease in traditional medicine ${ }^{13)}$. Sasang constitution diagnosis was measured using the K-prism Sasang constitution questionnaire ${ }^{18)}$. 
Twenty medical examinations were included as follows: 1) assessment of urine output after drinking, 2) chemical analysis of urine, 3) urine color assessment, 4) body temperature assessment, 5) assessment of skin moisture and skin lipids, 6) tongue-coating inspection, 7) halitosis assessment, 8) salivary flow rate assessment, 9) pulse assessment, 10) height/weight assessment, 11) body composition assessment, 12) heart rate variability assessment, 13) assessment of resting metabolic rate, 14) assessment of ventilation rate, 15) abdominal examination, 16) assessment of skin color, 17) K-prism Sasang constitution diagnosis, 18) 3D body scan, 19) posture assessment, 20) digital thermography.

The study was conducted at Kyung Hee University Korean Medicine Hospital, Pusan National University Korean Medicine Hospital, Naju Dongshin University Korean Medicine Hospital in 2019. This study received institutional review board approval from each of the three hospitals assessed (Kyung Hee: KOMCIRB 2019-10-003, Pusan: PNUKHIRB-009, and Dongshin: NJ-IRB-001).

\section{Participants}

A total of 90 subjects were recruited through posters, banners, and internet advertisements in and out of the hospital. The criteria for selection were (1) healthy men and women, (2) aged 19 years or older, and (3) had no cognitive impairment.

Exclusion criteria consisted of the following: (1) inability to move by themselves or measure the device; (2) had a current diagnosis of cardiovascular disease (e.g., myocardial infarction, congestive heart failure, angina, and arrhythmia), cerebrovascular disease (e.g., cerebral infarction, paralysis, etc.), malignant neoplasms (cancer), mental illness (depression, anxiety disorder), rheumatoid arthritis, thyroid disease (e.g., hyperthyroidism, hypothyroidism); (3) received medical treatment for internal medicine, nervous system diseases, and psychiatric disorders (except for high blood pressure, diabetes, and dyslipidemia) within the last month; (4) were pregnant, expecting to be pregnant, or breastfeeding; (5) were included in other studies within the past 30 days; (6) had any other condition that may affect their participation, based on the opinion of the investigators.

In addition, subjects were asked to avoid the following activities that could affect the medical examination: (1) excessive exercise within 3 days, (2) excessive diet control within 3 days, (3) smoking on the day, (4) caffeine intake on the day, (5) excessive drinking within 2 days, and (6) food intake within one hour.

All subjects eligible to participate in the study provided informed consent.

\section{Measures}

Subjects were screened for eligibility to participate in the study. Screening included demographic information, participant characteristics, medical history, medication use, and information about participation in other clinical trials. Participants were asked to answer 14 questionnaires and were examined with 20 medical examination devices. The primary outcome was the time required to complete each item of the KME. The total time taken to complete 10 clinical questionnaires and each of the 4 questionnaires 


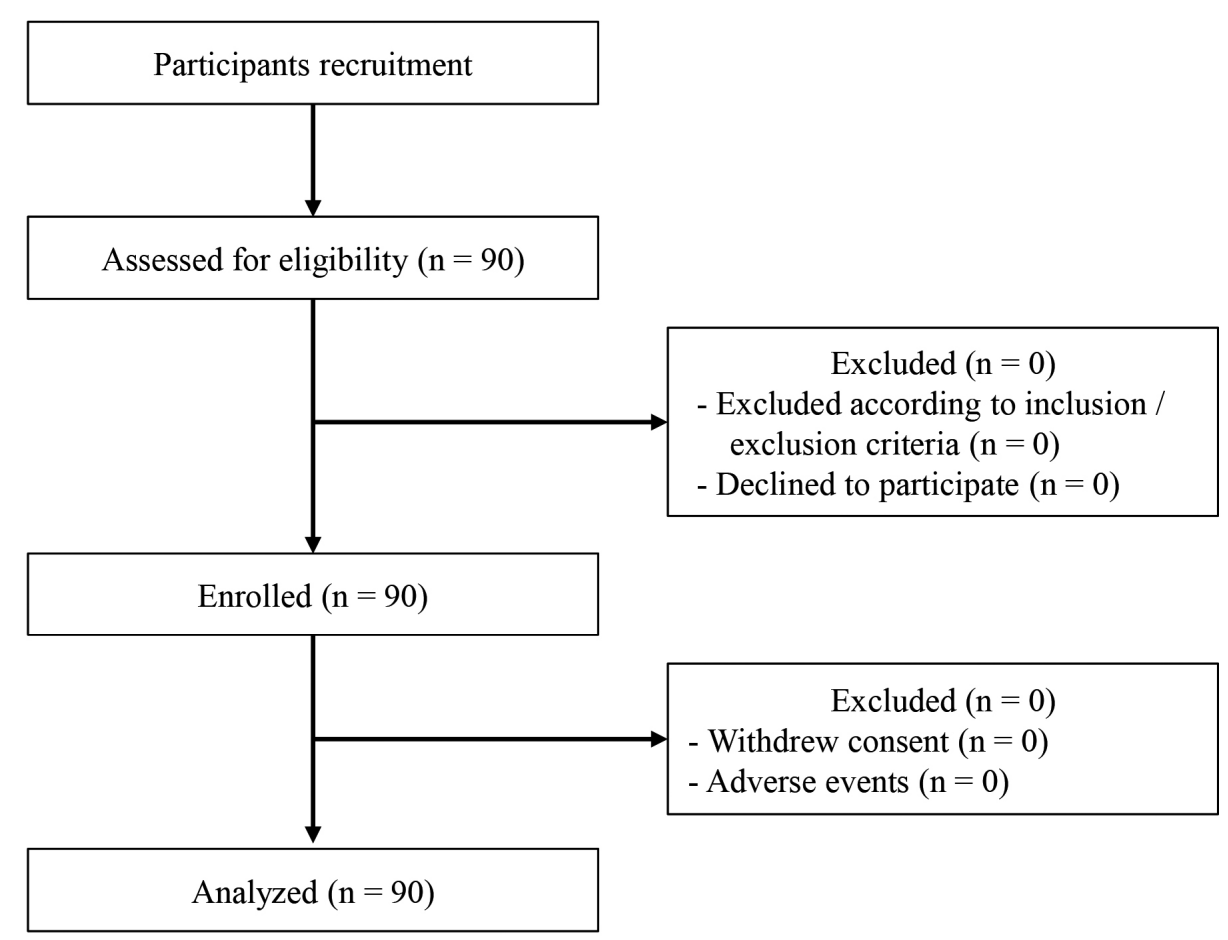

Fig. 1. Study flow chart

was measured in minutes. A research coordinator measured the time required to complete each medical examination procedure in minutes. The secondary outcome was satisfaction with the overall KME. After completing the KME, subjects completed a satisfaction survey on the following: 1) medical examinations they thought were most satisfactory (multiple responses available), 2) whether they intended to undergo a KME if hospitals provided them in the future, 3) the cost they thought was most appropriate for a KME, and 4) whether they intended to recommend a KME to others. Additionally, improvements in the KME protocol were proposed by the clinical coordinator and subjects. The safety, adverse events were assessed during the study.

\section{Statistical Analysis}

The mean and standardized deviation (SD) for the time spent on each KME program was assessed. All statistical analyses were performed using $\mathrm{R}$ version 3.6.2.

\section{Results}

A flow chart of enrollment is presented in Figure 1. The characteristics of the study participants are summarized in Table 1. Among the 90 participants, 31 (34.4\%) were men, and 59 (65.6\%) were women. The participant's age ranged from 19 to 64 years, and the average age was 37.2 (12.3) years. More than half (53.4\%) of the participants included were white-collar workers, 
Table 1. Characteristics of the Study Participants

$(n=90)$

\begin{tabular}{lr}
\hline Characteristics & $\mathrm{n}(\%)$ \\
\hline Sex & $31(34.4)$ \\
Male & $59(65.6)$ \\
Female & \\
\hline Age & $32(35.6)$ \\
$19-29$ & $27(30)$ \\
$30-39$ & $13(14.4)$ \\
$40-49$ & $11(12.2)$ \\
$50-59$ & $7(7.8)$ \\
$60-69$ & \\
\hline Occupation & $11(12.2)$ \\
Blue collar & $48(53.4)$ \\
White collar & $31(34.4)$ \\
Others & \\
Education & $0(0.0)$ \\
Below middle school graduate & $24(26.7)$ \\
High school graduate & $66(73.3)$ \\
Above college graduate &
\end{tabular}

and approximately three-quarters had more than a college degree.

Table 2 shows the average time spent on each item of the KME. The average time spent on the overall KME was approximately 88.0 (21.4) minutes. Approximately $33.5 \%$ of this time was used to complete the questionnaires and $66.5 \%$ for examination with the medical examination devices. The most time-consuming questionnaire was the CSEI (6.9 \pm 3.9 ), except for the 10 clinical questionnaires, and the most time-consuming medical examination device was the K-prism Sasang constitution diagnosis (10.1 \pm 4.2$)$.

The number of patients satisfied with the medical examination is shown in Table 3. The medical examinations with highest satisfaction scores included the 3D body scan $(n=42)$, body composition assessment $(\mathrm{n}=40)$, assessment of skin moisture and skin lipids $(\mathrm{n}=38)$, K-prism
Table 2. Average Time Spent on the Korean Medical Examination Program

\begin{tabular}{lc}
\hline & $\begin{array}{c}\text { Mean } \pm \text { SD } \\
\text { (Minutes) }\end{array}$ \\
\hline Total & $88.0 \pm 21.4$ \\
\hline Questionnaires & $29.5 \pm 13.8$ \\
10 Clinical questionnaires & $11.6 \pm 5.0$ \\
CSEI & $6.9 \pm 3.9$ \\
K-Prism Sasang constitution questionnaire & $4.7 \pm 2.5$ \\
PSQI & $3.9 \pm 3.0$ \\
PSS & $2.0 \pm 1.9$ \\
Medical examination & $58.6 \pm 17.3$ \\
K-prism Sasang constitution diagnosis & $10.1 \pm 4.2$ \\
3D body scan ${ }^{*}$ & $7.5 \pm 6.2$ \\
Halitosis assessment & $5.8 \pm 3.2$ \\
Salivary flow rate assessment & $5.8 \pm 0.8$ \\
Chemical analysis of urine & $4.7 \pm 3.9$ \\
Body composition assessment & $4.2 \pm 4.2$ \\
Pulse assessment & $4.0 \pm 2.8$ \\
Abdominal examination ${ }^{*}$ & $3.6 \pm 2.9$ \\
Assessment of skin color & $3.5 \pm 2.6$ \\
Digital thermography & $2.4 \pm 1.1$ \\
Heart rate variability assessment & $2.3 \pm 0.9$ \\
Assessment of skin moisture and skin lipids & $2.2 \pm 1.2$ \\
Urine color assessment ${ }^{*}$ & $2.2 \pm 3.4$ \\
Tongue-coating inspection & $0.4 \pm 0.5$ \\
Height/weight assessment & $0.3 \pm 0.5$ \\
Body temperature assessment & $0.3 \pm 0.5$ \\
\hline No response: 3D body scan ( $\mathrm{n}=2$ ), abdominal examination $(\mathrm{n}=7)$, \\
\hline
\end{tabular}

Sasang constitution diagnosis $(\mathrm{n}=35)$, and digital thermography $(\mathrm{n}=34)$.

Table 4 presents several indicators of satisfaction. Over $80 \%$ of study participants stated that they would be willing to receive $\mathrm{KME}$ if hospitals provide them and would recommend KME to others. Forty-three participants stated that the most appropriate cost for a KME was 100000 $199999 \mathrm{KRW}$, and 36 participants stated that the most appropriate was $<100000 \mathrm{KRW}$.

The areas for improvements provided by clinical coordinators are summarized in Table 5. 
Table 3. Number of Patients Satisfied with the Medical Examination using a Device $(n=90)$

\begin{tabular}{lc}
\hline Medical examination & $\mathrm{n}$ \\
\hline 3D body scan & 42 \\
Body composition assessment & 40 \\
Assessment of skin moisture and skin lipids & 38 \\
K-prism Sasang constitution diagnosis & 35 \\
Digital thermography & 34 \\
Pulse assessment & 28 \\
Heart rate variability assessment & 24 \\
Height/weight assessment & 22 \\
Halitosis assessment & 21 \\
Body temperature assessment & 15 \\
Assessment of skin color & 14 \\
Abdominal examination & 13 \\
Salivary flow rate assessment & 12 \\
Chemical analysis of urine & 9 \\
Tongue-coating inspection & 9 \\
Urine color assessment & 8 \\
\hline
\end{tabular}

There were three categories of improvements. First, questionnaire issues, including the number of items, understanding, and readability. The Core Seven Emotions Inventory consisted of 100 questions that might increase fatigue and decrease the reliability of participant responses. Some participants did not understand the questionnaire. In addition, the small size of the table and pictures provided in the questionnaire caused reading difficulties for the participants. Second, errors occurred during the medical examination for various reasons. The low stability of the device caused malfunctions in the 3D body scan. In addition, low compliance of participants during the halitosis assessment led to errors. Finally, the sequence of medical examinations was not optimized, and the temperature did not comply with standard operating procedures. Because each hospital had different environments, the order of medical examinations performed differed from the
Table 4. Satisfaction Survey $(n=90)$

\begin{tabular}{|c|c|}
\hline & $\mathrm{n}(\%)$ \\
\hline \multicolumn{2}{|c|}{ Intention to receive $\mathrm{KME}$ in the future } \\
\hline Yes & $74(82.2)$ \\
\hline No & $0(0)$ \\
\hline Don't know & $16(17.8)$ \\
\hline \multicolumn{2}{|c|}{ Intention to recommend $\mathrm{KME}$ to others* } \\
\hline Yes & $77(86.5)$ \\
\hline No & $0(0)$ \\
\hline Don't know & $12(13.5)$ \\
\hline \multicolumn{2}{|l|}{ Appropriate cost of a KME } \\
\hline$<100000 \mathrm{KRW}$ & $36(40.0)$ \\
\hline 100000 - $199999 \mathrm{KRW}$ & $43(47.8)$ \\
\hline 200000 - $299999 \mathrm{KRW}$ & $9(10.0)$ \\
\hline 300000 - $399999 \mathrm{KRW}$ & $1(1.1)$ \\
\hline 400000 - $499999 \mathrm{KRW}$ & $1(1.1)$ \\
\hline $500000 \mathrm{KRW}$ & $0(0)$ \\
\hline
\end{tabular}

order set by standard operating procedure. Participants felt cold, and although the appropriate temperature of the medical examination in the winter was suggested to be $18-20{ }^{\circ} \mathrm{C}$, the actual temperature in the room was maintained at 21-23 ${ }^{\circ} \mathrm{C}$.

The KME protocol was well tolerated, with no adverse events reported.

\section{Discussion}

This study aimed to evaluate the feasibility of the KME protocol in clinical practice. Through a literature review and expert group consensus, 14 questionnaires and 20 medical examinations were included in the KME protocol. Ninety participants with an average age of 37.2 years participated in the pilot study. It took approximately 88 minutes to perform the KME, including the 14 questionnaires and 20 medical examinations. Most 
participants were satisfied with the KME.

Suggestions for future research are as follows. First, the questionnaires provided to the subjects should be easy to understand and respond to. In this study, participants aged 59 years took 30 minutes to complete the CESI questionnaires. Because the CSEI has many items (100), it takes longer to answer the questions and is less reliable. Cheong et al. developed a short form of the CSEI scale (28 items) for use in clinical practice $^{19)}$. This is expected to shorten the response time by using only 28 items. In addition, a verification study will be conducted to confirm that the KME questionnaire tool accurately reflects the actual condition of the subject without error.

Second, efforts are needed to reduce device errors. In this study, except for the K-prism Sasang constitution diagnosis, the 3D body scan took the longest time among all medical device examinations. If the equipments works properly, measurements are usually obtained within 3 min; however, many malfunctions occurred due to the low stability of the device. Eventually, the time taken to scan the 3D body was longer due to needing 2-3 remeasurements, which took up to 35 minutes. To prevent these errors, dedicated personnel should be assigned at each site to immediately resolve device errors. Additionally, regular staff training would improve the work proficiency of the clinical coordinator.

Third, the results of various medical examinations should be provided to the subject. The subjects can understand the purpose of the medical examinations while checking their health conditions and increasing their satisfaction with the KME.

Fourth, because the KME protocol was not developed for early detection of specific diseases, it contains some items that do not have "reference values." In these cases, simply showing the measured values to the subject may not be meaningful as a medical examination. Therefore, to provide the meaning of the examination results to the subjects and utilize them in clinical practice and health management, research on reference data that can be used as a criterion for

Table 5. Types of Improvements Provided by the Clinical Coordinator at Each Hospital

\begin{tabular}{|c|c|c|}
\hline & Content & Example \\
\hline \multirow{3}{*}{ Questionnaires } & Number of Items & $\begin{array}{l}\text { Increasing fatigue and decreasing reliability of participants due to the } \\
\text { number of questions in the Core Seven Emotions Inventory }\end{array}$ \\
\hline & Understanding & Confusing 'menstrual cycle' as 'menstrual period' in menstrual health \\
\hline & Readability & $\begin{array}{l}\text { Unable to recognize table in eating habits and appearance of menstrual } \\
\text { bleeding pattern in menstrual health owing to the small size }\end{array}$ \\
\hline \multirow{3}{*}{$\begin{array}{l}\text { Error in medical } \\
\text { examination }\end{array}$} & Stability of device & Malfunction in 3D body scan due to the law stability of device \\
\hline & Compliance & Low compliance in halitosis assessment \\
\hline & Proficiency & $\begin{array}{l}\text { Insufficient proficiency resulting from many medical devices among the } \\
\text { small number of coordinators }\end{array}$ \\
\hline \multirow[b]{2}{*}{ Environment } & Examination sequence & Unoptimized sequence caused by different environments in each hospital \\
\hline & Temperature & $\begin{array}{l}\text { Participants felt the cold although the proper temperature of medical } \\
\text { examination in the winter was suggested to be } 18-20^{\circ} \mathrm{C} \text { and actual } \\
\text { temperature in the room was maintained at } 21-23^{\circ} \mathrm{C}\end{array}$ \\
\hline
\end{tabular}


judgment should also be conducted.

In conclusion, although this descriptive pilot study was conducted in 90 subjects in a short period of time, participants were highly satisfied with the KME. Future studies should address the issues raised in this pilot study so that accurate and rigorous $\mathrm{KME}$ data can be continuously collected.

\section{Acknowledgements}

This study was supported by the Korea Institute of Oriental Medicine (KSN2013110).

\section{References}

1. Mausner, J. S., Kramer, S., \& Bahn A. K. (1985). Epidemiology: An Introductory Text (2nd ed.). Philadelphia. Saunders.

2. National Health Insurance Service. (2018). 2018 National Health Screening Statistical Yearbook. Seoul. Available from: URL:https: //www.nhis.or.kr/nhis/together/wbhaec07000m0 1.do?mode $=$ view\&articleNo $=135196 \&$ article.of fset $=0$ \&articleLimit $=10$

3. Seong, S. C., Kim, Y. Y., Park, S. K., Khang, Y. H., Kim, H. C., Park, J. H., et al. (2017). Cohort profile: the national health insurance service-national health screening cohort (NHIS-HEALS) in Korea. BMJ open, 7(9), e016640. https://doi.org/10.1136/bmjopen-2017 $-016640$

4. Moon, J. S., Park, K. M., \& Choi, S. M. (2007). Study on the development of a questionnaire software for health examination in oriental medicine. Korean Journal of Oriental Medicine,
13(2), 135-142. https://www.koreascience.or.kr/ article/JAKO200736038246510.pdf

5. Chong, M. S., Kim, S. C., Lee, E. K., Chun, E. J., Han, J. M., Lee, S. K., et al. (2000). Research on the development of the oriental medical model on the health examination in the industry. Society of Preventive Korean Medicine, 4(1), 32-50. https://www.koreascience. or.kr/article/JAKO200004637423000.pdf

6. Park, Y. S., Kim, S. J., \& Kim, J. D. (2008). A study of the relationship between drinking, smoking and stress of workers by analyzing heart rate variability. Journal of Korean Medicine, 29(1), 134-145. https://www.koreascience. or.kr/article/JAKO200815750718906.pdf

7. Kwon, O. S., Kim, J. E., Lee, J. W., Seo, C. W., Han, H. Y., \& Hong, S. H. (2009). Analytic study of diagnostic validity by the measure of cold-heat \& deficiency-excess for oriental medical examination. Journal of Physiology \& Pathology in Korean Medicine, 23(1), 180-185. https://www.koreascience.or.kr/ article/JAKO200910335354145.pdf

8. Jung, H. J. \& Kim, S. M. (2013). A Relationship between Exercise and Health Factors including Stress, Fatigue and Vaso-aging degree. The Journal of the Korean Medicine Diagnostics, 17(2), 122-130. https://www.koreascience.or.kr/article/JAKO20 1301442489802.pdf

9. Lee, E. K., Jahng, D. S., Song, Y. S., \& Lee, K. N. (2002). The study on the contents and satisfaction of oriental medicine examination program. Society of Preventive Korean Medicine, 6(1), 51-95. https://www.koreascience. or.kr/article/JAKO200204637426993.pdf 
10. Han, H. J., Jeung, J. Y., Kwon, S. H., Song, Y. S., Jahng, D. S., \& Lee, K. N. (2003). Study on the satisfaction of oriental health examination for industrial workers. Journal of Korean Society of Occupational \& Environmental Hygiene, 13(2), 135-143. https://www.jksoeh.org/opensource/pdfjs/web/ pdf_viewer.htm?code $=\mathrm{J} 01302006$

11. Kim, H. H., Kim, J. Y., Park, Y. J., \& Park, Y. B. (2013). Development of pulse diagnostic devices in Korea. Integrative medicine research, 2(1), 7-17. https://doi.org/10.1016/j.imr.2013.01.003

12. Kim, J. H., Park, J. H., \& Kim, K. H. (2016). Review of Research topics on abdominal examination. Journal of Korean Medicine, 37(3), 1-12. https://doi.org/10.13048/jkm.16030

13. Lee, G. E., Park, B. Y., \& Moon, K. S., You, J. M., \& Kang, H. W. (2015). A study on the development of the Core Emotional Assessment Questionnaire (CEAQ) based on the seven emotions (七情). Journal of Oriental Neuropsychiatry, 26(2), 143-160. https://www.koreascience.or.kr/article/JAKO2 01521056138121.pdf

14. Buysse, D. J., Reynolds, C. F., Monk, T. H., Berman, S. R., \& Kupfer, D. J. (1989). The Pittsburgh Sleep Quality Index: a new instrument for psychiatric practice and research. Psychiatry research, 28(2), 193-213. https://doi.org/10.1016/0165-1781(89)90047-4

15. Sohn, S. I., Kim, D. H., Lee, M. Y., \& Cho, Y. W. (2012). The reliability and validity of the Korean version of the Pittsburgh Sleep Quality Index. Sleep Breath, 16(3), 803-812. https://doi.org/10.1007/s11325-011-0579-9
16. Cohen, S., Kamarck, T., \& Mermelstein, R. (1983). A global measure of perceived stress. Journal of health and social behavior, 24(4), 385-396. https://doi.org/10.2307/2136404

17. Lee, J. H., Shin, C. M., Ko, Y. H., Lim, J. H., Joe, S. H., Kim, S. H., et al. (2012). The reliability and validity studies of the Korean version of the Perceived Stress Scale. Korean Korean Journal of Psychosomatic Medicine, 20(2), 127-134. https://doi.org/10.3928/1940492120150806-72

18. Do, J. H., Jang, E. S., Ku, B. C., Jang, J. S., Kim, H. G., \& Kim, J. Y. (2012). Development of an integrated Sasang constitution diagnosis method using face, body shape, voice, and questionnaire information. BMC complementary and alternative medicine, 12(1), 85. https://doi.org/10.1186/1472-6882-12-85

19. Cheong, M. J., Lee, G. E., Lee, Y. S., Bae, K. H., Kang, Y. S., Kim, J. H., et al. (2019). Validation of the Core Seven-Emotions Inventory-short form. Integrative medicine research, 8(2), 116-119. https://doi.org/10.1016/ j.imr.2019.04.003

\section{ORCID}

Junghun Yoo https://orcid.org/0000-0002-0980-6364 Sanghun Lee https://orcid.org/0000-0001-7124-6874 Soyoung Kim https://orcid.org/0000-0002-5245-6515 Daehyeok Kim https://orcid.org/0000-0003-2160-1377 Jeong Hwan Park https://orcid.org/0000-0003-2697-6530 